\title{
LETTER
}

\section{First principles prediction of new high-pressure phase of InOOH}

\author{
Jun Tsuchiya*, Taku TsuchiYA ${ }^{*}$, Asami SANo ${ }^{* *}$ and Eiji OHTANI ${ }^{* * *}$ \\ *Geodynamics Research Center, Ehime University, 2-5 Bunkyo-cho, \\ Matsuyama, Ehime 790-8577, Japan \\ **Institute for solid state physics, University of Tokyo, 5-1-5 Kashiwanoha, \\ Kashiwa, Chiba 277-8581, Japan \\ *** Department of Mineralogy, Petrology, and Economic Geology, Tohoku University, \\ Aoba Aramaki Aza, Sendai 980-8578, Japan
}

\begin{abstract}
We have predicted a new high-pressure phase of $\mathrm{InOOH}$ by first principles density functional calculations, which stabilizes at $\sim 15 \mathrm{GPa}$ under the static $0 \mathrm{~K}$ condition. The structure obtained at $15 \mathrm{GPa}$ has a pyrite-type $\mathrm{InO}_{2}$ framework with the interstitial asymmetric hydrogen bonds, which is assigned to the space group $P 2_{1} 3$ (No. 198). The cell parameters and internal atomic coordinates optimized at $15 \mathrm{GPa}$ are $a=5.265 \AA, \mathrm{In}_{x}=0.005$, $\mathrm{O} 1_{x}=0.367, \mathrm{O} 2_{x}=0.633$, and $\mathrm{H}_{x}=0.487$. Relative enthalpies also indicate that the dehydration of this phase into $\mathrm{In}_{2} \mathrm{O}_{3}+\mathrm{H}_{2} \mathrm{O}$ is unlikely to occur at least up to $50 \mathrm{GPa}$ under the static condition. To our knowledge, this is the first report on the high-pressure isochemical phase transition of oxyhydroxide compounds to the pyrite-type structure. The new phase of $\mathrm{InOOH}$ has been identified in an experiment, as reported in detail in a separate paper. We have also found that the low-pressure-type asymmetric hydrogen bond in the new pyrite-type phase changes to the symmetric hydrogen bond over $30 \mathrm{GPa}$. The present results suggest that a similar phase relation is expected in other group IIIB oxyhydroxides such as $\mathrm{AlOOH}$ and $\mathrm{GaOOH}$.
\end{abstract}

Keywords: InOOH, First principles, High pressure, Hydrogen bond

\section{INTRODUCTION}

$\delta$ - $\mathrm{AlOOH}$ is a high-pressure phase of $\mathrm{AlOOH}$ (diaspore) that has recently been found at $\sim 21 \mathrm{GPa}$ and $1000{ }^{\circ} \mathrm{C}$ (Suzuki et al., 2000). The $\delta^{-} \mathrm{AlOOH}$ phase has the $\mathrm{CaCl}_{2}{ }^{-}$ type structure (Fig. 1a) and is found to be stable under the $P$ and $T$ conditions inferred for cold slabs subducting into the deep mantle (Ohtani et al., 2001). Due to the high stability of this phase under high pressure, it is proposed to be a possible carrier and reservoir of water in the deep Earth (Sano et al., 2004). Therefore, its high-pressure structural behaviors have attracted considerable geophysical interest. In particular, it is interesting to determine whether this phase remains stable, decomposes into an anhydrous phase and $\mathrm{H}_{2} \mathrm{O}$, or transforms into a higherpressure hydrous phase at the Earth's lower mantle pressures.

We have recently reported pressure-induced hydrogen bond symmetrizations in $\delta$ - $\mathrm{AlOOH}$ and phase $\mathrm{D}$

doi:10.2465/jmps.071022e

J.Tsuchiya, junt@sci.ehime-u.ac.jp Corresponding author
( $\left.\mathrm{MgSi}_{2} \mathrm{O}_{6} \mathrm{H}_{2}\right)$ occurring above $\sim 30$ and $\sim 40 \mathrm{GPa}$, respectively (Tsuchiya et al., 2002, 2005). Since among the known hydrous minerals, only these two phases are reported to be stable at lower mantle pressures (Ohtani et al., 2001; Sano et al., 2004), it is presumable that the hydrogen bond symmetrization stabilizes hydrous minerals under high pressure. However, the stability limit and further high-pressure structural evolution of these phases have not been well understood.

Considering the above-mentioned facts, we first focus on the high-pressure behavior of $\mathrm{InOOH}$ since $\mathrm{In}^{3+}$ is homologous to $\mathrm{Al}^{3+}$ and $\mathrm{InOOH}$ can be expected to behave as a low-pressure analog of $\mathrm{AlOOH}$. InOOH has the $\mathrm{CaCl}_{2}$-type structure, even under ambient conditions; this structure is isostructural to $\delta^{-} \mathrm{AlOOH}$. Therefore, a highpressure phase of $\mathrm{InOOH}$, if found, would suggest a new high-pressure phase transition of $\delta^{-} \mathrm{AlOOH}$. The studies of analog materials are meaningful and helpful, particularly for high-pressure experiments. Since the transition pressures expected in $\mathrm{InOOH}$ are less than those expected in $\mathrm{AlOOH}$, the study will increase the feasibility of the 

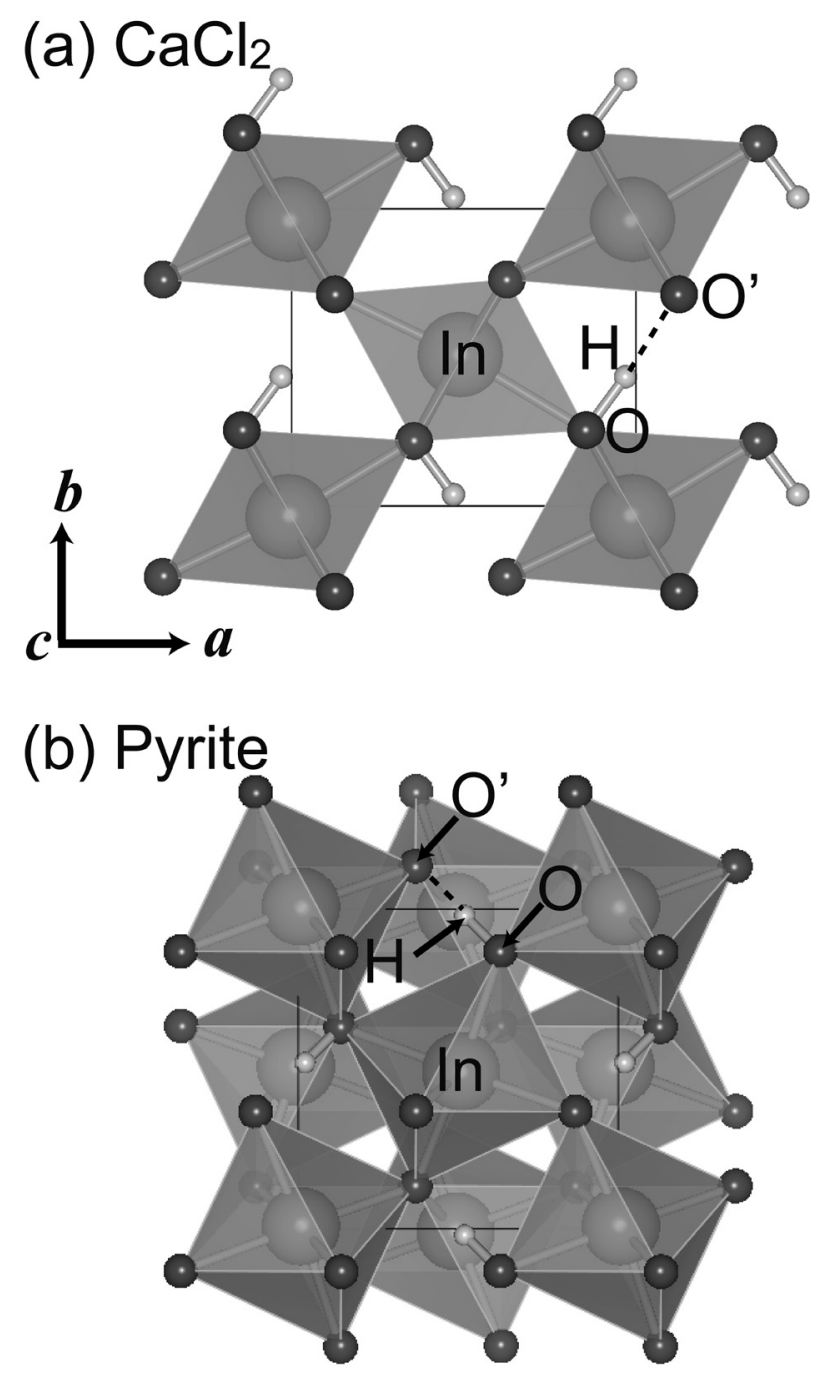

Figure 1. Crystal structures of (a) $\mathrm{CaCl}_{2}$ and (b) pyrite-type InOOH. Large, medium, and small spheres denote In, $\mathrm{O}$, and $\mathrm{H}$ atoms, respectively.

experimental corroboration of the transitions (Sano et al., 2008).

The $\mathrm{CaCl}_{2}$-type structure has been reported in several $M^{3+} \mathrm{OOH}$ oxyhydroxide compounds $(M=\mathrm{Al}, \mathrm{Sc}, \mathrm{V}, \mathrm{Cr}$, Mn, Fe, Ni, Rh, and In) (Chenavas et al., 1973; Baneyeva and Bendeliani, 1973; Suzuki et al., 2000). However, to the best of our knowledge, there is no report on the highpressure structure transition of these $\mathrm{CaCl}_{2}$-type oxyhydroxides. In this study, we explore the new phase relation of $\mathrm{InOOH}$ using the first principles density functional techniques by testing the energy balance of several structure models which are potentially possible to exist as post- $\mathrm{CaCl}_{2}$ oxyhydroxide.

\section{CALCULATION METHODS AND STRUCTURE MODELS}

Our computations are based on the density functional theory with the generalized gradient approximation (GGA) to the exchange-correlation functional (Perdew et al., 1996). The GGA is critical for the accurate simulation of the weak hydrogen bond (Hamann, 1997). Pseudopotentials (Troullier and Martins, 1991; Rappe et al., 1990) are applied to describe the ionic core potentials of $\mathrm{O}\left(2 s^{2}\right.$ and $\left.2 p^{4}\right), \mathrm{H}\left(1 s^{1}\right)$, and $\mathrm{In}\left(4 d^{10}, 5 s^{2}\right.$, and $\left.5 p^{1}\right)$. The electronic wave functions are expanded by plane waves with an energy cutoff of 80 Ry $(1 \mathrm{Ry}=13.6058 \mathrm{eV})$. We calculate the following four structures of $\mathrm{InOOH}$ : orthorhombic diaspore structure, $\mathrm{CaCl}_{2}$ structure, $\alpha-\mathrm{PbO}_{2}$ structure, and cubic pyrite structure. In addition, anhydrous $\operatorname{In}_{2} \mathrm{O}_{3}$ and $\mathrm{H}_{2} \mathrm{O}$ are computed to investigate the possible high-pressure dehydration reaction. In the pressure range considered in this study $(\mathrm{P}<50 \mathrm{GPa}), \mathrm{In}_{2} \mathrm{O}_{3}$ and $\mathrm{H}_{2} \mathrm{O}$ are reported to crystallize into orthorhombic $\mathrm{Rh}_{2} \mathrm{O}_{3}$ (II) and tetragonal ice VIII structures, respectively (Yusa et al., 2007; Song et al. 2003). The irreducible parts of the Brillouin zones of the diaspore, $\mathrm{CaCl}_{2}, \alpha-\mathrm{PbO}_{2}$, pyrite-type InOOH, $\mathrm{Rh}_{2} \mathrm{O}_{3}$ (II)-type $\mathrm{In}_{2} \mathrm{O}_{3}$ and ice VIII structures are sampled on $1 \times 4 \times 2,2 \times 2 \times 4,4 \times 2 \times 2,4 \times 4 \times 4,4 \times$ $4 \times 4$ and $4 \times 4 \times 4$ Monkhorst-Pack meshes (Monkhorst and Pack, 1976), respectively. The effects of larger energy cutoffs and $k$-point samplings on the calculated properties are found to be insignificant. All structural parameters are fully relaxed to static $(0 \mathrm{~K})$ configurations using the damped variable cell shape molecular dynamics method (Wentzcovitch, 1991) implemented with the PWscf code (http://www.pwscf.org) until residual forces become less than $1.0 \times 10^{-5}$ Ry/au.

The major part of this study involves the construction of high-pressure model structures of post- $\mathrm{CaCl}_{2}$-type InOOH. We have constructed these structures crystallographically as follows. The $\mathrm{CaCl}_{2}$-type structure can be adapted to the high-pressure form of group IVB dioxides, $A \mathrm{O}_{2}(A=\mathrm{Si}, \mathrm{Ge}, \mathrm{Sn}$, and $\mathrm{Pb})$. This suggests that the highpressure phase relation in $\mathrm{InOOH}$ might also follow the high-pressure phase sequence of group IVB dioxides. In the case of $\mathrm{SiO}_{2}$, the $\mathrm{CaCl}_{2}$ phase has been reported to transform into $\alpha-\mathrm{PbO}_{2}$ structure at $\sim 100 \mathrm{GPa}$ and further into the pyrite phase above $200 \mathrm{GPa}$ (e.g., Tsuchiya et al., 2004b; Kuwayama et al., 2005). Therefore, we construct the post- $\mathrm{CaCl}_{2}$ model structures that have the $\alpha-\mathrm{PbO}_{2}$ and pyrite-type $\mathrm{InO}_{6}$ octahedral frameworks with hydrogen atoms in their interstitial spaces. In addition to the $\mathrm{InOOH}$ polymorphs, we test the possible stability of the dehydration of InOOH into $\mathrm{Rh}_{2} \mathrm{O}_{3}$ (II)-type $\mathrm{In}_{2} \mathrm{O}_{3}$ and ice VIII, as mentioned earlier. 


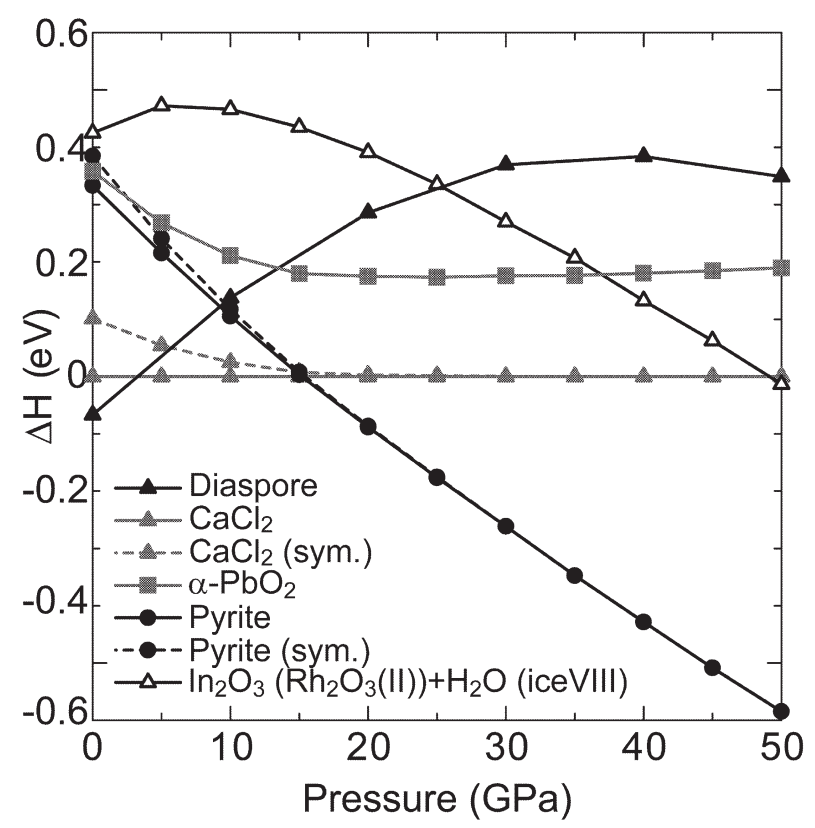

Figure 2. Relative enthalpies of InOOH polymorphs with respect to $\mathrm{CaCl}_{2}$-type phase. Dashed lines indicate the low-pressure metastable symmetric hydrogen bond states of $\mathrm{CaCl}_{2}$ and pyrite-type structures.

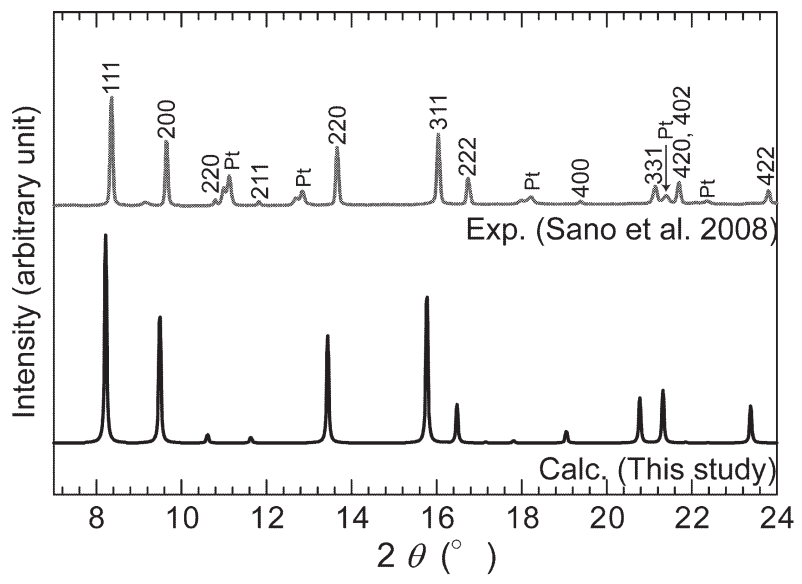

Figure 4. Calculated and experimental (Sano et al. 2008) X-ray $(\lambda$ $=0.4266 \AA$ ) diffraction patterns of pyrite type InOOH obtained at $30 \mathrm{GPa}$.

\section{RESULTS AND DISCUSSION}

All the structural parameters of the initial model structures are first fully relaxed at pressures from 0 to $50 \mathrm{GPa}$ under the static $0 \mathrm{~K}$ condition. Figure 2 shows the calculated static enthalpies of the model structures relative to the $\mathrm{CaCl}_{2}$-type structure as a function of pressure. Diaspore type structure is found to be more stable than $\mathrm{Ca}$ $\mathrm{Cl}_{2}$-type structure at 0-3 $\mathrm{GPa}$, although the experiments report that $\mathrm{CaCl}_{2}$-type structure is stable under ambient conditions (Christensen et al., 1964). This discrepancy is

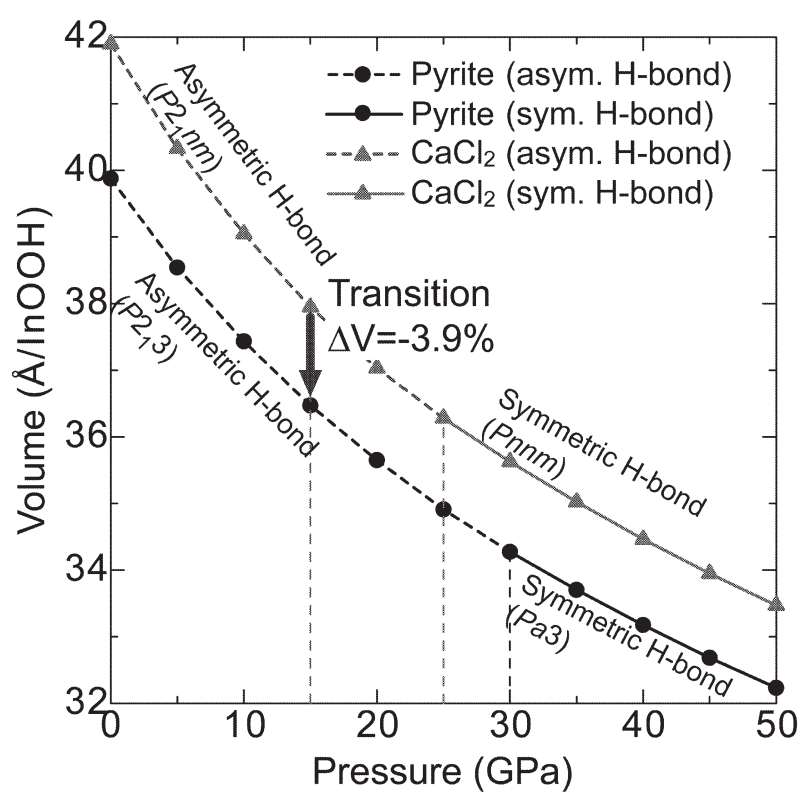

Figure 3. Equations of state of the pyrite (circles) and $\mathrm{CaCl}_{2}$-type (triangles) phases. Solid and dashed lines indicate the symmetric and asymmetric hydrogen bond states, respectively.

due to the general tendency of the GGA to overestimate (typically by 5-10 GPa) the stability range of low-pressure structures (e.g., Tsuchiya et al., 2004a). With increasing pressure, an enthalpy crossover appears at $\sim 15 \mathrm{GPa}$, where the pyrite structure becomes more stable than $\mathrm{Ca}$ $\mathrm{Cl}_{2}$-type structure. To our knowledge, this is the first report on the high-pressure isochemical phase transition of oxyhydroxide compounds to the pyrite-type structure. Although $\alpha-\mathrm{PbO}_{2}$ is reported to be the post $-\mathrm{CaCl}_{2}$ phase in group VIB dioxides, the enthalpy of $\alpha-\mathrm{PbO}_{2}$ structure is significantly higher than that of pyrite structure in $\mathrm{InOOH}$. This indicates that there is no stability field for $\alpha-\mathrm{PbO}_{2}$ in $\mathrm{InOOH}$. The higher lattice energy of $\alpha-\mathrm{PbO}_{2}$-type $\mathrm{InOOH}$ is probably because of the very small hydrogen-hydrogen distances $(\sim 2.78 \AA$ ), which are estimated by the hydrogen positions in this structure. Moreover, the calculated enthalpy of $\mathrm{Rh}_{2} \mathrm{O}_{3}$ (II)-type $\operatorname{In}_{2} \mathrm{O}_{3}+$ ice VIII is considerably higher than that of pyrite-type $\mathrm{InOOH}$, even at $50 \mathrm{GPa}$. This means that the dehydration reaction is thermodynamically unfavorable at least under static $0 \mathrm{~K}$ condition.

Figure 3 shows the equations of state of the $\mathrm{CaCl}_{2}$ and pyrite-type phases. The zero-pressure bulk moduli (pressure derivatives) for the $\mathrm{CaCl}_{2}$ and pyrite type phases are determined to be $109.5 \mathrm{GPa}$ (6.47) and $128.2 \mathrm{GPa}$ (5.82), respectively, by the least-square fitting of the $P-V$ relation to the Vinet equation (Vinet et al., 1989). A volume reduction of $\sim 3.9 \%$ is expected for the transition from the $\mathrm{CaCl}_{2}$ to pyrite at $15 \mathrm{GPa}$ (Fig. 3). Pyrite-type InOOH has been successfully identified by complementary high-pressure experiments (Fig. 4). The results will be 
Table 1. Crystal structures of the $\mathrm{CaCl}_{2}$ and pyrite-type $\mathrm{InOOH}$

\begin{tabular}{llllll}
\hline & $\mathrm{CaCl}_{2}$ & \multicolumn{5}{c}{ Pyrite } \\
\hline $\mathrm{P}(\mathrm{GPa})$ & 0 & 30 & 0 & 15 & 30 \\
$\mathrm{~S} . \mathrm{G}$. & $P{ }_{1} n m$ & $P n n m$ & $P 2{ }_{1} 3$ & $P 2{ }_{1} 3$ & $P a \overline{3}$ \\
$a(\AA)$ & 5.381 & 5.136 & 5.423 & 5.265 & 5.156 \\
$b$ & 4.649 & 4.367 & - & - & - \\
$c$ & 3.349 & 3.176 & - & - & - \\
$\mathrm{In}(x, y, z)$ & $(0,0.227,0)$ & $(0,0,0)$ & $(0.010,0.010,0.010)$ & $(0.005,0.005,0.005)$ & $(0,0,0)$ \\
$\mathrm{O}(x, y, z)$ & $(0.366,0.482,0)$ & $(0.365,0.219,0)$ & $(0.365,0.365,0.365)$ & $(0.367,0.367,0.367)$ & $(0.367,0.367,0.367)$ \\
$\mathrm{O}(x, y, z)$ & $(0.636,0.025,0)$ & - & $(0.633,0.633,0.633)$ & $(0.633,0.633,0.633)$ & - \\
$\mathrm{H}(x, y, z)$ & $(0.480,0.302,0)$ & $(0.5,0,0)$ & $(0.477,0.477,0.477)$ & $(0.487,0.487,0.487)$ & $(0.5,0.5,0.5)$ \\
$\mathrm{H}-$ bond & Asymmetric & Symmetric & Asymmetric & Asymmetric & Symmetric \\
\hline
\end{tabular}

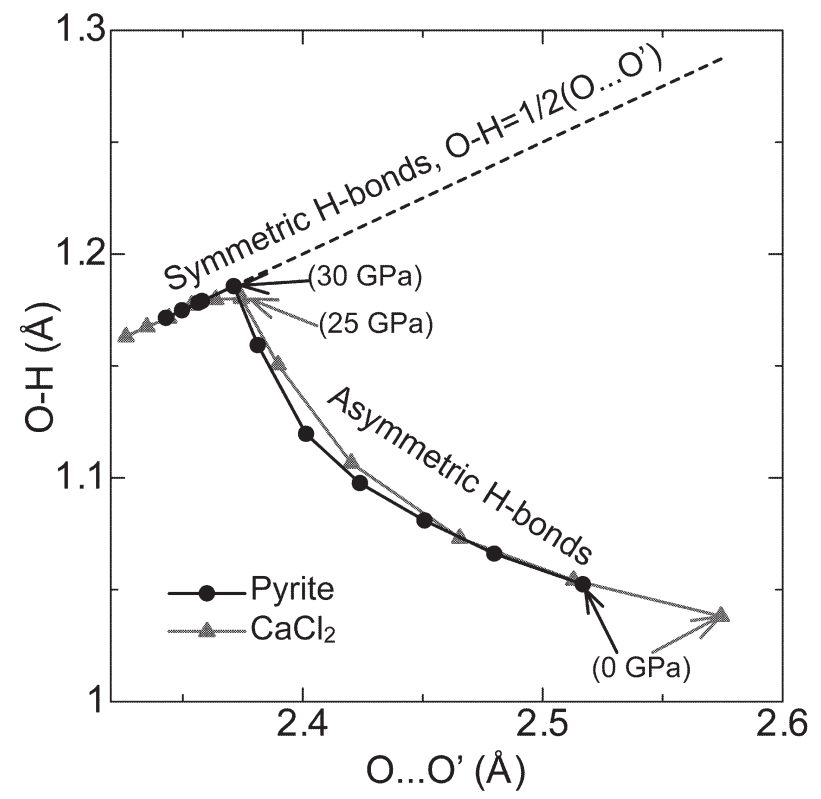

Figure 5. Relationships between $\mathrm{O} \ldots \mathrm{O}^{\prime}$ and $\mathrm{O}-\mathrm{H}$ distances of $\mathrm{CaCl}_{2}$ (triangles) and pyrite-type (circles) structures. Pressure interval between adjacent dots and triangles is $5 \mathrm{GPa}$, and longest and shortest $\mathrm{O} \ldots \mathrm{O}^{\prime}$ lengths corresponds to lowest and highest pressures of 0 and $50 \mathrm{GPa}$, respectively. Dashed line represents the distance corresponding to $\mathrm{O}-\mathrm{H}=1 / 2\left(\mathrm{O} \ldots \mathrm{O}^{\prime}\right)$.

reported in detail separately (Sano et al., 2008). The most stable hydrogen positions in these structures are determined by the structure optimization with the calculation of the total energies of trial structures in which hydrogen atoms are placed at several vacant sites between two oxygen atoms in the interstitials of the $\mathrm{InO}_{6}$ octahedra. Then, we have found the most stable hydrogen positions with the lowest energy in the $\mathrm{CaCl}_{2}$ and pyrite structures. They have the asymmetric hydrogen bonds at $0 \mathrm{GPa}$, which cause a slight distortion of $\mathrm{InO}_{6}$ octahedron. Therefore, the structures are less symmetric than the ideal $\mathrm{CaCl}_{2}$ and pyrite structures. The $\mathrm{CaCl}_{2}$ and pyrite structures at $0 \mathrm{GPa}$ can be assigned to the space groups $P 2_{1} n m$ (No. 31) and
$P 2{ }_{1} 3$ (No. 198), respectively (Table 1).

In our previous study, the pressure-induced hydrogen bond symmetrization in $\delta$ - $\mathrm{AlOOH}$ was reported to occur at $\sim 30 \mathrm{GPa}$ (Tsuchiya et al., 2002), which was achieved by the continuous displacement of hydrogen atoms from the asymmetric off-centered position between two neighboring oxygen atoms $\left(\mathrm{O}\right.$ and $\mathrm{O}^{\prime}$ in Fig. 1$)$ to the symmetric centered position. We have carefully determined the hydrogen positions of $\mathrm{InOOH}$ polymorphs and found that the symmetric hydrogen bonds are formed in the $\mathrm{CaCl}_{2}$ and pyrite-type structures at 25 and $30 \mathrm{GPa}$, respectively. In relation to these changes in the hydrogen bonds, the crystallographic symmetries of the $\mathrm{CaCl}_{2}$ and pyrite phases increase to the space groups Pnnm and $P a \overline{3}$, respectively (Table 1).

Figure 5 shows the relationships between the distance between two neighboring oxygen atoms $\left(\mathrm{O} \ldots \mathrm{O}^{\prime}\right)$ and the bond length of oxygen and hydrogen atoms $(\mathrm{O}-\mathrm{H})$ in the $\mathrm{CaCl}_{2}$ and pyrite structures (Fig. 1). In both the structures, the $\mathrm{O}-\mathrm{H}$ lengths first increase under compression and approach half the value of the $\mathrm{O} \ldots \mathrm{O}^{\prime}$ distances. This indicates the strengthening of the hydrogen bond and the weakening of the $\mathrm{O}-\mathrm{H}$ bond. Then, the symmetrizations are achieved at an $\mathrm{O} \ldots \mathrm{O}^{\prime}$ distance of $\sim 2.37 \AA$ in both the $\mathrm{CaCl}_{2}$ and pyrite structures. After the hydrogen bond symmetrization, the $\mathrm{O}-\mathrm{H}$ lengths begin to decrease along the $\mathrm{O}-\mathrm{H}=1 / 2\left(\mathrm{O} \ldots \mathrm{O}^{\prime}\right)$ line. It should be noted that the relationship between the $\mathrm{O}-\mathrm{H}$ lengths and $\mathrm{O} \ldots \mathrm{O}^{\prime}$ distances appears to be independent of the structure type. In the present study, we neglect both the quantum and thermal vibration effects of the light hydrogen atoms. These effects are expected to decrease the pressures of the hydrogen bond symmetrization (Benoit et al., 1999).

\section{CONCLUSIONS}

We have reported new high-pressure phase relations in indium oxyhydroxide $(\mathrm{InOOH})$ predicted by first princi- 
ples calculations. Pyrite-type $\mathrm{InOOH}$ is found to stabilize above $\sim 15 \mathrm{GPa}$ instead of the $\mathrm{CaCl}_{2}$-type phase. No dehydration reaction is observed at least up to $50 \mathrm{GPa}$ under athermal condition; the $\alpha-\mathrm{PbO}_{2}$-type phase also is observed to be unstable. Calculations indicate that the $\mathrm{CaCl}_{2}$ and pyrite-type structures have the symmetric hydrogen bonds over 25 and $30 \mathrm{GPa}$, respectively. Similar transitions from the $\mathrm{CaCl}_{2}$ to pyrite-type phase are expected in other group IIIB oxyhydroxides such as $\mathrm{AlOOH}$ and $\mathrm{GaOOH}$, although their transition pressure would be higher than that of $\mathrm{InOOH}$.

\section{ACKNOWLEDGMENTS}

JT is grateful to the JSPS Fellowship for Young Scientists. The calculations were performed using a GRC parallel computing system. This study was partly supported by a Grant-in-Aid for Scientific Research from the Japan Society for the Promotion of Science (Grants Nos. 18840033 and 19740331).

\section{REFERENCES}

Baneyeva, M.I. and Bendeliani, N.A. (1973) The $\mathrm{Fe}_{2} \mathrm{O}_{3}-\mathrm{H}_{2} \mathrm{O}$ system at high pressures and extemperatures. Geochemistry International, 10, 840-842.

Benoit, M., Marx, D. and Parrinello, M. (1999) The role of quantum effects and ionic defects in high-density ice. Solid State Ionics, 125, 23-29.

Chenavas, J., Joubert, J.C., Capponi, J.J. and Marezio, M. (1973) Synthese de Nouvelles Phase Denses D'oxyhydroxydes $\mathrm{M}^{3+} \mathrm{OOH}$ des Metaux de la Premiere Serie de Transition, en Milieu Hydrothermal à Tres Haute Pression. Journal of Solid State Chemistry, 6, 1-15.

Christensen, A.N., Gronbaek, R. and Rasmussen, S.E. (1964) Crystal structure of InOOH. Acta Chemica Scandinavica, 18, 1261-1266.

Hamann, D.R. (1997) $\mathrm{H}_{2} \mathrm{O}$ hydrogen bonding in density-functional theory. Physical Review B, 55, R10157-R10160.

Kuwayama, Y., Hirose, K., Sata, N. and Ohishi, Y. (2005) The pyrite-type high-pressure form of silica. Science, 309, 923-925.

Monkhorst, H.J. and Pack, J.D. (1976) Special points for Brillouin-zone integrations. Physical Review B, 13, 5188-5192.

Ohtani, E., Litasov, K., Suzuki, A. and Kondo, T. (2001) Stability field of new hydrous phase, $\delta-\mathrm{AlOOH}$, with implications for water transport into deep mantle. Geophysical Research Let- ters, 28, 3991-3993

Perdew, J.P., Burke, K. and Ernzerhof, M. (1996) Generalized gradient approximation made simple. Physical Review Letters, 77, 3865-3868.

Rappe, A.M., Rabe, K.M., Kaxiras, E. and Joannopoulous, J.D. (1990) Optimized pseudopotentials. Physical Review B, 41, R1227-R1230

Sano, A., Ohtani, E., Kubo, T. and Funakoshi, K. (2004) In situ $\mathrm{X}$-ray observation of decomposition of hydrous aluminum silicate $\mathrm{AlSiO}_{3} \mathrm{OH}$ and aluminum oxide hydroxide d-AlOOH. Journal of Physics and Chemistry of Solids, 65, 1547-1554.

Sano, A., Yagi, T., Okada, T., Gotou, H., Ohtani, E., Tsuchiya, J. and Kikegawa, T. (2008) X-ray diffraction study of high pressure transition in InOOH. Journal of Mineralogical and Petrological Sciences, 103, 152-155.

Song, M., Yamawaki, H., Fujihisa, H., Sakashita, M. and Aoki, K. (2003) Infrared investigation on ice VIII and the phase diagram of dense ices. Physical Review B, 68, 014106.

Suzuki, A., Ohtani, E. and Kamada, T. (2000) A new hydrous phase $\delta-\mathrm{AlOOH}$ synthesized at $21 \mathrm{GPa}$ and $1000^{\circ} \mathrm{C}$. Physics and Chemistry of Minerals, 27, 689-693.

Troullier, N. and Martins, J.L. (1991) Efficient pseudopotential for plane wave calculations, Physical Review B, 43, 1993-2006.

Tsuchiya, J., Tsuchiya, T., Tsuneyuki, S. and Yamanaka, T. (2002) First principles calculation of a high-pressure hydrous phase, $\delta$-AlOOH. Geophysical Research Letters, 29, 1909.

Tsuchiya, T., Tsuchiya, J., Umemoto, K. and Wentzcovitch, R.M. (2004a) Phase transition in $\mathrm{MgSiO}_{3}$ perovskite in the earth's lower mantle. Earth and Planetary Science Letters, 224, 241-248.

Tsuchiya, T., Caracas, R. and Tsuchiya, J. (2004b) First principles determination of the phase boundaries of high-pressure polymorphs of silica. Geophysical Research Letters, 31, L11610.

Tsuchiya, J., Tsuchiya, T. and Tsuneyuki, S. (2005) First-principles study of hydrogen bond symmetrization of phase D under high pressure. American Mineralogist, 90, 44-49.

Vinet, P., Rose, J.H., Ferrante, J. and Smith, J.R. (1989) Universal features of the equation of state of solids, Journal of Physics: Condensed Matter, 1, 1941-1963.

Wentzcovitch, R.M. (1991) Invariant molecular dynamics approach to structural phase transitions. Physical Review B, 44, 2358-2361.

Yusa, H., Tsuchiya, T., Sata, N. and Ohishi, Y. (2007) $\mathrm{Rh}_{2} \mathrm{O}_{3}(\mathrm{II})^{-}$ type structures in $\mathrm{Ga}_{2} \mathrm{O}_{3}$ and $\mathrm{In}_{2} \mathrm{O}_{3}$ under high pressure. Physical Review B, 77, 064107.

Manuscript received October 22, 2007

Manuscript accepted December 18, 2007

Manuscript handled by Kiyoshi Fujino 